\title{
Connectivity of Matching graph of Hypercube
}

\author{
Jiří Fink* \\ Department of Applied Mathematics \\ Faculty of Mathematics and Physics \\ Charles University \\ Malostranské náměstí 25 \\ 11800 Prague 1 \\ E-mail: fink@kam.mff.cuni.cz
}

\begin{abstract}
The matching graph $\mathcal{M}(G)$ of a graph $G$ has a vertex set of all perfect matchings of $G$, with two vertices being adjacent whenever the union of the corresponding perfect matchings forms a Hamiltonian cycle.

We prove that the matching graph $\mathcal{M}\left(Q_{d}\right)$ of the $d$-dimensional hypercube is bipartite and connected for $d \geq 4$. This proves Kreweras' conjecture [8] that the graph $M_{d}$ is connected, where $M_{d}$ is obtained from $\mathcal{M}\left(Q_{d}\right)$ by contracting all vertices of $\mathcal{M}\left(Q_{d}\right)$ which correspond to isomorphic perfect matchings.
\end{abstract}

\section{Introduction}

A set of edges $P \subseteq E$ of a graph $G=(V, E)$ is a matching if every vertex of $G$ is incident with at most one edge of $P$. If a vertex $v$ of $G$ is incident with an edge of $P$, then $v$ is covered by $P$, otherwise $v$ is uncovered by $P$. A matching $P$ is perfect if every vertex of $G$ is covered by $P$.

The $d$-dimensional hypercube (shortly $d$-cube) $Q_{d}$ is a graph whose vertex set consists of all binary vectors of length $d$, with two vertices being adjacent whenever the corresponding vectors differ at exactly one coordinate. The binary vectors are labelled by the set $[d]:=$ $\{1,2, \ldots, d\}$.

It is well known that $Q_{d}$ is Hamiltonian for every $d \geq 2$. This statement can be traced back to 1872 [7]. Since then the research on Hamiltonian cycles in $d$-cubes satisfying certain additional properties has received considerable attention. An interested reader can find more details about this topic in the survey of Savage [10]. Dvořák [3] showed that every set of at most $2 d-3$ edges of $Q_{d}(d \geq 2)$ that induces vertex-disjoint paths is contained in a Hamiltonian cycle. Dimitrov et al. [1] proved that for every perfect matching $P$ of $Q_{d}(d \geq 3)$ there exists some Hamiltonian cycle that faults $P$ if and only if $P$ is not a set of all edges of one dimension of $Q_{d}$.

*This work was partially supported by the Czech Science Foundation 201/05/H014 and the Czech Ministry of Education 1M0545. 
The matching graph $\mathcal{M}(G)$ of a graph $G$ on an even number of vertices has a vertex set of all perfect matchings of $G$, with two vertices being adjacent whenever the union of the corresponding perfect matchings forms a Hamiltonian cycle of $G$; e.g. Figure 1 shows the matching graph $\mathcal{M}(G)$. There is a natural one-to-one correspondence between Hamiltonian cycles of $G$ and edges of $\mathcal{M}(G)$. The problem of determining $h(d)$, the number of Hamiltonian cycles of a $d$-cube, is a well-known open problem. Douglas [2] presents upper and lower bounds

$$
\left(\prod_{i=5}^{d-1} i^{2^{d-i-1}}\right) d(1344)^{2^{d-4}} 2^{2^{d-2}-1-d} \leq h(d) \leq\left(\frac{d(d-1)}{2}\right)^{2^{d-1}-2^{d-1-\log _{2}(d)}} .
$$

We are interested in structural properties of $\mathcal{M}\left(Q_{d}\right)$.

We say that two perfect matchings $P$ and $R$ are isomorphic if there exists an isomorphism $f: V\left(Q_{d}\right) \rightarrow V\left(Q_{d}\right)$ such that $f(u) f(v) \in R$ for every edge $u v \in P$. This relation of isomorphism is an equivalence and it partitions the set of all perfect matchings. Kreweras [8] considered a graph $M_{d}$ which is obtained from $\mathcal{M}\left(Q_{d}\right)$ by contracting all vertices of each class of this equivalence. For example, $Q_{3}$ has two non-isomorphic perfect matchings, so $M_{3}$ has two vertices connected by an edge. The graph $M_{4}$ is presented on Figure 3.

Kreweras [8] proved by inspection of all perfect matchings that the graphs $M_{3}$ and $M_{4}$ are connected and he conjectured that the graph $M_{d}$ is connected for every $d \geq 3$. It is more general to also ask whether the graph $\mathcal{M}\left(Q_{d}\right)$ is connected since the connectivity of $\mathcal{M}\left(Q_{d}\right)$ implies the connectivity of $M_{d}$. The answer is negative for $d=3$ (see Figure 1). However, we prove that this is the only counter-example.
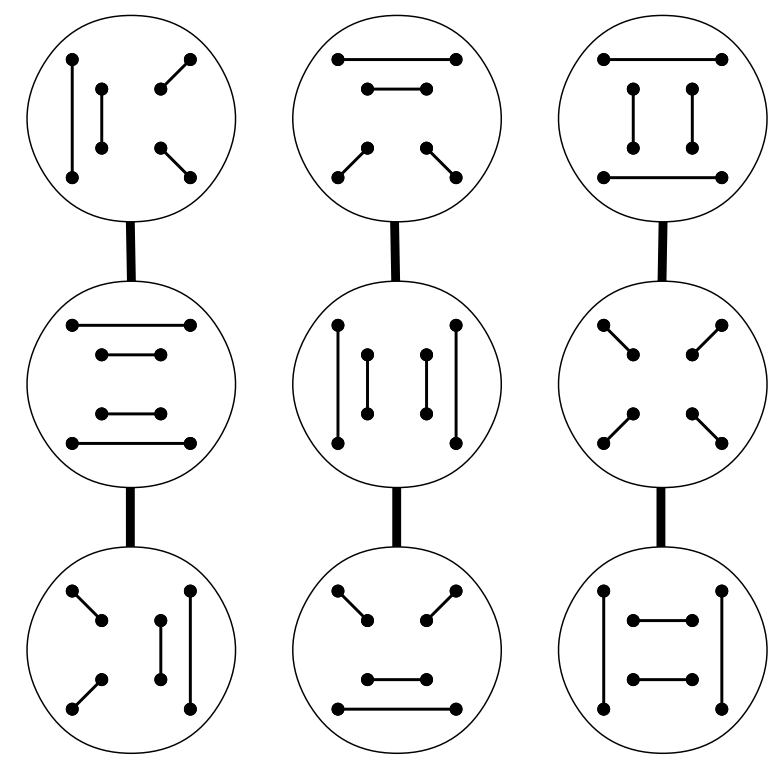

Figure 1: The matching graph $\mathcal{M}\left(Q_{3}\right)$. The circles and bold lines are vertices and edges of $\mathcal{M}\left(Q_{3}\right)$.

We also prove that the matching graph $\mathcal{M}\left(K_{n, n}\right)$ of the complete bipartite graph $K_{n, n}$ is bipartite for even $n$, which implies that $\mathcal{M}\left(Q_{d}\right)$ is bipartite. This is an interesting property which helps us find a walk in $\mathcal{M}\left(Q_{d}\right)$ of even length. We show other interesting properties of matching graphs $\mathcal{M}\left(Q_{d}\right)$ and $\mathcal{M}\left(K_{n, n}\right)$ in [4]. 


\section{Perfect matchings extend to Hamiltonian cycles}

Let $K(G)$ be the complete graph on the vertices of a graph $G$. If $G$ is bipartite and connected, then let $B(G)$ be a complete bipartite graph with the same color classes as $G$. Let $P$ be a perfect matching of $K\left(Q_{d}\right)$. Let $\Gamma(P)$ be the set of all perfect matchings $R$ of $Q_{d}$ such that $P \cup R$ is a Hamiltonian cycle of $K\left(Q_{d}\right)$. Note that if $P$ is a perfect matching of $Q_{d}$ and $R \in \Gamma(P)$, then $P \cup R$ is a Hamiltonian cycle of $Q_{d}$, so $P R$ is an edge of $\mathcal{M}\left(Q_{d}\right)$.

Kreweras conjectured [8] that every perfect matching in the $d$-cube with $d \geq 2$ extends to a Hamiltonian cycle. We [5] proved the following stronger form of this conjecture.

Theorem 1 (Fink [5]). For every perfect matching $P$ of $K\left(Q_{d}\right)$ the set $\Gamma(P)$ is non-empty where $d \geq 2$.

We say that an edge $u v$ of $K\left(Q_{d}\right)$ crosses a dimension $\alpha \in[d]$ if vertices $u$ and $v$ differ in dimension $\alpha$, otherwise $u v$ avoids $\alpha$. A perfect matching $P$ of $K\left(Q_{d}\right)$ crosses $\alpha$ if $P$ contains an edge crossing $\alpha$, otherwise $P$ avoids $\alpha$. Let $I_{d}^{\alpha}$ be the perfect matching of $Q_{d}$ that contains all edges in dimension $\alpha \in[d]$. Observe that a perfect matching $P$ of $Q_{d}$ crosses $\alpha$ if and only if $P \cap I_{d}^{\alpha} \neq \emptyset$.

Proposition 2. Let $P$ be a perfect matching of $K\left(Q_{d}\right)$ avoiding $\beta \in[d]$ and $e \in I_{d}^{\beta}$. There exists $R \in \Gamma(P)$ containing e.

Proof. The proof proceeds by induction on $d$. The statement holds for $d=2$. Let us assume that the statement is true for every $k$-cube $Q_{k}$ with $2 \leq k \leq d-1$ and let us prove it for $d$.

Clearly, $P$ crosses some $\alpha \in[d] \backslash\{\beta\}$. We divide the $d$-cube $Q_{d}$ by dimension $\alpha$ into two $(d-1)$-subcubes $Q^{1}$ and $Q^{2}$ so that $e \in E\left(Q^{1}\right)$. Let $K^{i}:=K\left(Q^{i}\right)$ and $P^{i}:=P \cap E\left(K^{i}\right)$ for $i \in\{1,2\}$.

The set of edges $P^{1}$ is a matching of $K^{1}$ which is not perfect since $P$ crosses $\alpha$. Let $M$ be the set of vertices of $K^{1}$ that are uncovered by $P^{1}$. The size of $M$ is even. If we divide $Q^{1}$ by dimension $\beta$, then numbers of vertices of $M$ on both subcubes of $Q^{1}$ are even because $P^{1}$ avoids $\beta$. We choose an arbitrary perfect matching $S^{1}$ on vertices of $M$ such that $S^{1}$ avoids $\beta$. The perfect matching $P^{1} \cup S^{1}$ of $K^{1}$ avoids $\beta$. By induction there exists a perfect matching $R^{1} \in \Gamma\left(P^{1} \cup S^{1}\right)$ of $Q^{1}$ containing $e$. Let

$$
S^{2}:=\left\{x y \in E\left(K^{2}\right) \mid \begin{array}{c}
\exists x^{\prime}, y^{\prime} \in V\left(Q^{1}\right) \text { such that } x x^{\prime}, y y^{\prime} \in P \text { and } \\
\text { there exists a path between } x^{\prime} \text { and } y^{\prime} \text { of } P^{1} \cup R^{1}
\end{array}\right\} .
$$

Observe that $P^{1} \cup R^{1}$ is a partition of $Q^{1}$ into vertex-disjoint paths between vertices uncovered by $P^{1}$. For every path between $x^{\prime}$ and $y^{\prime}$ of this partition there exist vertices $x$ and $y$ of $Q^{2}$ such that $x x^{\prime}, y y^{\prime} \in P$. Thus, the set of edges $S^{2}$ is a matching of $K^{2}$. Moreover, the set of edges $P^{2} \cup S^{2}$ is a perfect matching of $K^{2}$ because $S^{2}$ covers each vertex covered by $P$ but not by $P^{2}$. Hence, there exists a perfect matching $R^{2} \in \Gamma\left(P^{2} \cup S^{2}\right)$ of $Q^{2}$ by Theorem 1 . Clearly, $R:=R^{1} \cup R^{2}$ is a perfect matching of $Q_{d}$ containing $e$. Finally, $R \in \Gamma(P)$ by Lemma 3 .

Lemma 3. Let $P$ be a perfect matching of $K\left(Q_{d}\right)$ crossing some dimension $\alpha \in[d]$. Let the $d$-cube $Q_{d}$ be divided into two $(d-1)$-subcubes $Q^{1}$ and $Q^{2}$ by dimension $\alpha$. Let $K^{i}:=K\left(Q^{i}\right)$ and $P^{i}:=P \cap E\left(K^{i}\right)$ for $i \in\{1,2\}$. Let $S^{1}$ be a perfect matching on vertices of $K\left(Q^{1}\right)$ uncovered by $P^{1}$. Let $R^{1} \in \Gamma\left(P^{1} \cup S^{1}\right)$. Let $S^{2}$ be given by $(1)$. Let $R^{2} \in \Gamma\left(P^{2} \cup S^{2}\right)$ and $R:=R^{1} \cup R^{2}$. Then $R \in \Gamma(P)$. 
Proof. We prove that $P \cup R$ is a Hamiltonian cycle of $K\left(Q_{d}\right)$. Suppose on the contrary that $C$ is a cycle of $P \cup R$ which is not Hamiltonian. Since $P$ crosses $\alpha$, both $S^{1}$ and $S^{2}$ are non-empty sets. Because $P^{i} \cup S^{i} \cup R^{i}$ is a Hamiltonian cycle of $K^{i}$, whole cycle $C$ cannot belong to $K^{i}$, for $i \in\{1,2\}$. So $C$ has edges in both $K^{1}$ and $K^{2}$. Now, we shorten every path $x x^{\prime} \cdots y^{\prime} y$ such that $x, y \in V\left(Q^{2}\right) ; x^{\prime}, y^{\prime} \in V\left(Q^{1}\right) ; x x^{\prime}, y y^{\prime} \in P$ and $x^{\prime} \cdots y^{\prime}$ is a path of $P^{1} \cup R^{1}$ by the edge $x y \in S^{2}$. Hence, we obtain a cycle $C^{\prime}$ of $\left(P^{2} \cup S^{2}\right) \cup R^{2}$. We prove that $C^{\prime}$ does not contain a vertex of $K^{2}$ which is a contradiction because $\left(P^{2} \cup S^{2}\right) \cup R^{2}$ is a Hamiltonian cycle of $K^{2}$.

If $C$ does not contain a vertex $u$ of $K^{2}$, then $C^{\prime}$ also does not contain $u$. Suppose that $C$ does not contain a vertex $v$ of $K^{1}$. Let $x^{\prime}$ and $y^{\prime}$ be the end vertices of the longest path of $P^{1} \cup R^{1}$ that contains $v$. Let $x x^{\prime}, y y^{\prime} \in P$. Observe that $x, y \in V\left(K^{2}\right)$ and $x y \in S^{2}$. Hence, $C^{\prime}$ does not contain $x$ and $y$.

Observe that the perfect matching $R$ obtained in Lemma 3 avoids dimension $\alpha$. The interested reader may ask whether there exists a perfect matching $R$ in Theorem 1 that avoids given set of dimension $A \subset[d]$. Clearly, the graph on edges of $P$ and allowed edges of $Q_{d}$ (i.e. edges of $Q_{d}$ that avoid every dimension of $A$ ) must be connected. Gregor [6] proved that this is also a sufficient condition which implies following lemma.

Lemma 4. For every perfect matching $P$ of $K\left(Q_{d}\right)$ and $\alpha \in[d]$ there exists $R \in \Gamma(P)$ avoiding $\alpha$ if and only if $P$ crosses $\alpha$ where $d \geq 2$.

Moreover, Ruskey and Savage [9, page 19, question 3] asked the following more general question:

Does every (not necessarily perfect) matching of $Q_{d}$ for $d \geq 2$ extend to a Hamiltonian cycle of $Q_{d}$ ?

\section{Bipartiteness of $\mathcal{M}\left(K_{n, n}\right)$}

There is a natural one-to-one correspondence between perfect matchings of the complete bipartite graph $K_{n, n}$ and permutations on a set of size $n$. A permutation $\pi$ is even if $n-k$ is even where $k$ is a number of cycles of $\pi$, otherwise $\pi$ is odd. It is well-known that $\pi_{1} \circ \pi_{2}$ is even if and only if permutations $\pi_{1}$ and $\pi_{2}$ have the same parity. Hence, the inverse permutation $\pi_{2}^{-1}$ has the same parity as $\pi_{2}$.

Let $c(P)$ be the number of components of the graph on a set of edges $P$. Recall that $B(G)$ is the complete bipartite graph with the same color classes as a bipartite and connected graph $G$.

Let $P_{1}$ and $P_{2}$ be perfect matchings of $K_{n, n}$ and $\pi_{1}$ and $\pi_{2}$ be their corresponding permutations. Observe that $c\left(P_{1} \cup P_{2}\right)$ is equal to the number of cycles of $\pi_{1} \circ \pi_{2}^{-1}$. If $n$ is even and $P_{1} \cup P_{2}$ is a Hamiltonian cycle of $K_{n, n}$, then $\pi_{1}$ and $\pi_{2}$ have different parities. Hence, $\mathcal{M}\left(K_{n, n}\right)$ is bipartite for $n$ even. The matching graph $\mathcal{M}\left(Q_{d}\right)$ is also bipartite because $\mathcal{M}\left(Q_{d}\right)$ is a subgraph of $\mathcal{M}\left(B\left(Q_{d}\right)\right)$ which is isomorphic to $\mathcal{M}\left(K_{2^{d-1}, 2^{d-1}}\right)$.

The above discussion proves the following theorem.

Theorem 5. The matching graphs $\mathcal{M}\left(Q_{d}\right)$ and $\mathcal{M}\left(B\left(Q_{d}\right)\right)$ are bipartite. 
We did not define which perfect matchings of $B\left(Q_{d}\right)$ are even and odd. But we know that perfect matchings $P_{1}$ and $P_{2}$ of $B\left(Q_{d}\right)$ belong to the same color class of $\mathcal{M}\left(B\left(Q_{d}\right)\right)$ if and only if $c\left(P_{1} \cup P_{2}\right)$ is even. Hence, we fix one perfect matching of $B\left(Q_{d}\right)$ to be even.

Let us recall that $I_{d}^{\alpha}$ is the perfect matching of $Q_{d}$ that contains all edges in dimension $\alpha \in[d]$. We simply count that $c\left(I_{d}^{\alpha} \cup I_{d}^{\beta}\right)=2^{d-2}$ for every two different dimensions $\alpha, \beta \in[d]$ because the graph on edges $I_{d}^{\alpha} \cup I_{d}^{\beta}$ consists of $2^{d-2}$ independent cycles of size 4 . Hence, perfect matchings $I_{d}^{\alpha}$ and $I_{d}^{\beta}$ belong to the same color class of $\mathcal{M}\left(B\left(Q_{d}\right)\right)$ for $d \geq 3$. We call a perfect matching $P$ of $B\left(Q_{d}\right)$ even if $c\left(P \cup I_{d}^{1}\right)$ is even and otherwise odd where $d \geq 3$.

\section{Walks in $\mathcal{M}\left(Q_{d}\right)$}

We will prove that $\mathcal{M}\left(Q_{d}\right)$ is connected by induction on $d$. Therefore, we need to know how we can make a walk in $\mathcal{M}\left(Q_{d}\right)$ from a walk in $\mathcal{M}\left(Q_{d-1}\right)$. In this section we present two lemmas which help us.

Let $P^{0}$ and $P^{1}$ be perfect matchings of $Q_{d-1}$. We denote by $\left\langle P^{0} \mid P^{1}\right\rangle$ the perfect matching of $Q_{d}$ containing $P^{i}$ in the $(d-1)$-subcube of vertices having $i$ in the coordinate $d$ for $i \in\{0,1\}$.

Lemma 6. Let $P_{1}, P_{2}, P_{3}, R_{1}, R_{2}$, and $R_{3}$ be perfect matchings of $Q_{d-1}$ such that $P_{1} \cup P_{2}$, $P_{2} \cup P_{3}, R_{1} \cup R_{2}$, and $R_{2} \cup R_{3}$ are Hamiltonian cycles of $Q_{d-1}$. If $P_{2} \cap R_{2} \neq \emptyset$, then there exists a perfect matching $S$ of $Q_{d}$ such that $\left\langle P_{1} \mid R_{1}\right\rangle \cup S$ and $S \cup\left\langle P_{3} \mid R_{3}\right\rangle$ are Hamiltonian cycles of $Q_{d}$. Moreover, $S$ crosses the dimension $d$ and every dimension that is crossed by $P_{2}$ or $R_{2}$.

Proof. Let $u v \in P_{2} \cap R_{2}$. Let $u_{i}$ be the vertex of $Q_{d}$ obtained from $u$ by appending $i$ into dimension $d$, where $i \in\{0,1\}$. Vertices $v_{0}$ and $v_{1}$ are defined similarly.

Let $S:=\left(\left\langle P_{2} \mid R_{2}\right\rangle \backslash\left\{u_{0} v_{0}, u_{1} v_{1}\right\}\right) \cup\left\{u_{0} u_{1}, v_{0} v_{1}\right\}$. The graph on edges $\left\langle P_{1} \mid R_{1}\right\rangle \cup\left\langle P_{2} \mid R_{2}\right\rangle$ consists of two cycles covering all vertices of $Q_{d}$. These cycles are joined together in $\left\langle P_{1} \mid R_{1}\right\rangle \cup$ $S$. Hence, $\left\langle P_{1} \mid R_{1}\right\rangle \cup S$ is a Hamiltonian cycle of $Q_{d}$. Similarly, $S \cup\left\langle P_{3} \mid R_{3}\right\rangle$ is a Hamiltonian cycle of $Q_{d}$.

The edge $u_{0} u_{1}$ crosses dimension $d$, so $S$ also crosses $d$. Let us consider a dimension $\beta \in[d-1]$ which is crossed by $P_{2}$ or $R_{2}$. Without loss of generality we suppose that $P_{2}$ crosses $\beta$. There exist at least 2 edges crossing $\beta$ in $P_{2}$. It can happen that the edge $u_{0} v_{0}$ is one of them, so at least one edge crossing $\beta$ remains in $S$.

Let $P$ be a perfect matching of $K\left(Q_{d}\right)$ and $A \subseteq[d]$. We say that $P$ crosses $A$ if $P$ crosses every dimension of $A$.

Lemma 7. Let $P_{1}, P_{2}, P_{3}$, and $R_{1}$ be perfect matchings of $Q_{d-1}$ such that $P_{1} \cup P_{2}$ and $P_{2} \cup P_{3}$ are Hamiltonian cycles of $Q_{d-1}$. Let $\alpha, \beta \in[d-1], \alpha \neq \beta$. If $P_{2}$ crosses $[d-1] \backslash\{\alpha\}$ and $R_{1}$ avoids $\beta$, then there exists a perfect matching $S$ of $Q_{d}$ such that $\left\langle P_{1} \mid R_{1}\right\rangle \cup S$ and $S \cup\left\langle P_{3} \mid R_{1}\right\rangle$ are Hamiltonian cycles of $Q_{d}$ and $S$ crosses $[d] \backslash\{\alpha\}$.

Proof. Let $e \in P_{2} \cap I_{d-1}^{\beta}$. There exists $R_{2} \in \Gamma\left(R_{1}\right)$ containing $e$ by Proposition 2 . If we apply Lemma 6 on $P_{1}, P_{2}, P_{3}, R_{1}, R_{2}$, and $R_{1}$, then we obtain a perfect matching $S$ which satisfies the requirements of this lemma. 


\section{Base of induction}

Let us recall that $M_{d}$ is obtained from $\mathcal{M}\left(Q_{d}\right)$ by contracting all vertices of $\mathcal{M}\left(Q_{d}\right)$ whose corresponding perfect matchings are isomorphic. Let $P$ and $R$ be perfect matchings of $Q_{d}$. If there exists a walk between vertices representing $P$ and $R$ in $\mathcal{M}\left(Q_{d}\right)$, then the length of the shortest one is $d(P, R)$, otherwise $d(P, R)$ is infinity. Hence, $d(P, R)<\infty$ means that $P$ and $R$ belong to the same component of $\mathcal{M}\left(Q_{d}\right)$.

The proof, that $\mathcal{M}\left(Q_{d}\right)$ is connected, proceeds by induction on $d$. We present a base of this induction in this section. We showed that $\mathcal{M}\left(Q_{3}\right)$ has 3 components (see Figure 1), so the induction starts from $d=4$. Kreweras [8] proved that $M_{4}$ is connected (see Figure 3). We prove that if $M_{d}$ is connected and $d \geq 4$, then $\mathcal{M}\left(Q_{d}\right)$ is connected. Hence, $\mathcal{M}\left(Q_{4}\right)$ is connected.

First, we present a simple lemma.

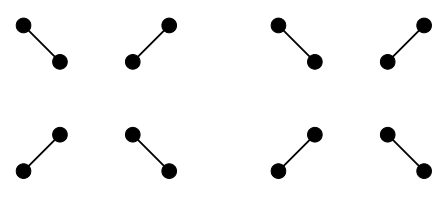

Perfect matching $S_{4}^{0}=I_{4}^{\alpha}$

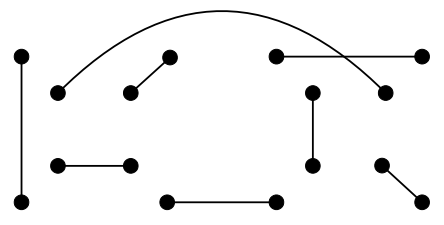

Perfect matching $S_{4}^{3}$

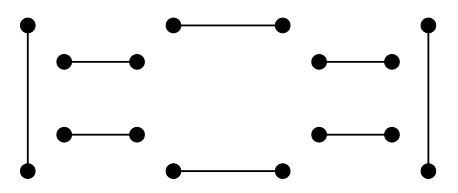

Perfect matching $S_{4}^{1}$

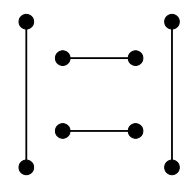

Perfect matching $S_{4}^{4}$

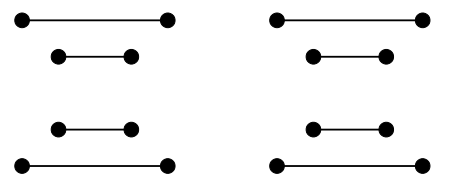

Perfect matching $S_{4}^{6}=I_{4}^{\beta}$

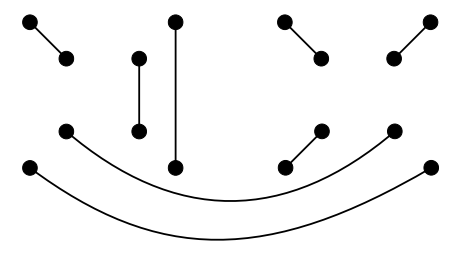

Perfect matching $S_{4}^{2}$

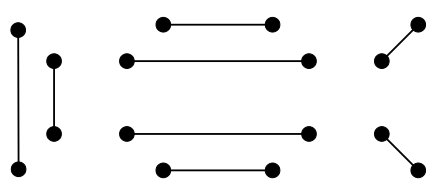

Perfect matching $S_{4}^{5}$

Figure 2: The walk between perfect matchings $I_{4}^{\alpha}$ and $I_{4}^{\beta}$ in $\mathcal{M}\left(Q_{4}\right)$.

Lemma 8. If $d \geq 4$, then $d\left(I_{d}^{\alpha}, I_{d}^{\beta}\right) \leq 6$ for every $\alpha, \beta \in[d], \alpha \neq \beta$.

Proof. The proof proceeds by induction on $d$. The walk between $I_{4}^{\alpha}$ and $I_{4}^{\beta}$ is drawn in Figure 2.

Let $I_{d-1}^{\alpha}=S_{d-1}^{0}, S_{d-1}^{1}, S_{d-1}^{2}, S_{d-1}^{3}, S_{d-1}^{4}, S_{d-1}^{5}, S_{d-1}^{6}=I_{d-1}^{\beta}$ be a walk in $\mathcal{M}\left(Q_{d-1}\right)$. Let $S_{d}^{i}:=\left\langle S_{d-1}^{i} \mid S_{d-1}^{i}\right\rangle$ for even $i$. For odd $i$ let $S_{d}^{i}$ be given by Lemma 6 where $P_{1}=R_{1}:=S_{d-1}^{i-1}$, $P_{2}=R_{2}:=S_{d-1}^{i}$, and $P_{3}=R_{3}:=S_{d-1}^{i+1}$. Then $I_{d}^{\alpha}=S_{d}^{0}, S_{d}^{1}, S_{d}^{2}, S_{d}^{3}, S_{d}^{4}, S_{d}^{5}, S_{d}^{6}=I_{d}^{\beta}$ is a walk in $\mathcal{M}\left(Q_{d}\right)$. 
Let us recall that perfect matchings $P$ and $R$ are isomorphic if there exists an isomorphism $f: V\left(Q_{d}\right) \rightarrow V\left(Q_{d}\right)$ such that $f(u) f(v) \in R$ for edge $u v \in P$. This relation of isomorphism is an equivalence on the set of all perfect matching. Let $[P]$ be the equivalence class containing $P$. Observe that $\left[I_{d}\right]:=\left\{I_{d}^{\alpha} \mid \alpha \in[d]\right\}$ is an equivalence class. If there exists a walk between $[P]$ and $[R]$ of $M_{d}$, then the length of the shortest one is $d([P],[R])$, otherwise $d([P],[R])$ is infinity.

Let us consider perfect matchings $P$ and $R$ of $Q_{d}$ such that $d([P],[R])=1$. There exist isomorphisms $f$ and $g$ such that $f(P) \cup g(R)$ forms a Hamiltonian cycle. Moreover, $P \cup f^{-1}(g(R))$ forms a Hamiltonian cycle. Hence, we have a perfect matching $f^{-1}(g(R)) \in$ $\Gamma(P)$ such that $f^{-1}(g(R))$ is isomorphic to $R$.

Proposition 9. If $d \geq 4$ and $M_{d}$ is connected, then $\mathcal{M}\left(Q_{d}\right)$ is connected.

Proof. We prove that vertices $\left\{P \in V\left(\mathcal{M}\left(Q_{d}\right)\right) \mid d\left([P],\left[I_{d}\right]\right) \leq k\right\}$ belong into one component of $\mathcal{M}\left(Q_{d}\right)$ by induction on $k$. This claim holds for $k=0$ by Lemma 8 .

Let $P$ be a perfect matching of $Q_{d}$ such that $d\left([P],\left[I_{d}\right]\right)=k$. There exists a perfect matching $R$ of $Q_{d}$ such that $d\left([R],\left[I_{d}\right]\right)=k-1$ and $d([P],[R])=1$. Hence, there exists $R^{\prime} \in \Gamma(P)$ isomorphic to $R$. By induction $d\left(I_{d}, R^{\prime}\right)<\infty$. Therefore, $d\left(P, I_{d}\right)<\infty$.

Type 4400, number 12

Type 6200 , number 48

Type 4400, number 24

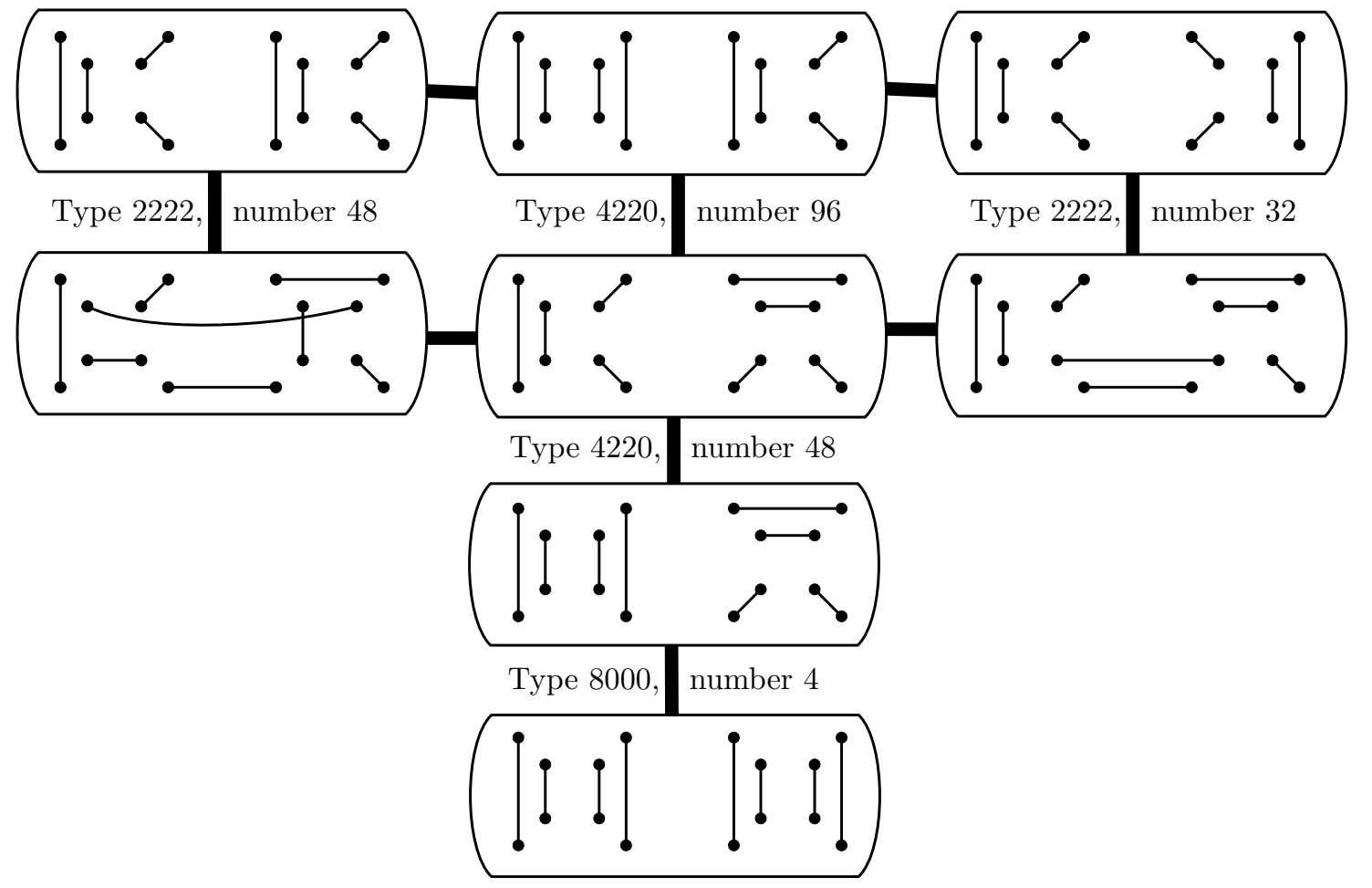

Figure 3: The graph $M_{4}$. For every equivalence class $[P]$ of isomorphism there is a frame which contains $P$. Four type numbers above each frame are numbers of edges crossing each dimension. Above each frame there is also a number of perfect matchings which are contracted to the equivalence class. 


\section{Induction step}

We define a set of perfect matchings $\mathcal{Z}(d, k, \alpha)$ of $Q_{d}$ by following induction on $d$, where $d \geq k \geq 3$ and $\alpha \in[d]$.

Definition 10. Let $\mathcal{Z}(d, d, \alpha)$ contain only $I_{d}^{\alpha}$. The set $\mathcal{Z}(d, k, \alpha)$, where $d>k \geq 3$ and $\alpha \in[d]$, is the set of all perfect matchings of $Q_{d}$ in the form $\left\langle P_{1} \mid P_{2}\right\rangle$, where $P_{1} \in \mathcal{Z}(d-1, k, \alpha)$ and $P_{2}$ is an even perfect matching of $Q_{d-1}$ avoiding some dimension $\beta \in[d-1] \backslash\{\alpha\}$.

Observe that every perfect matching of $\mathcal{Z}(d, k, \alpha)$ is even and it contains $I_{k}^{\alpha}$ in some $k$ subcube $Q_{k}$. We want to prove that the graph $\mathcal{M}\left(Q_{d}\right)$ is connected, so we need to show that there exists a perfect matching $I$ of $Q_{d}$ such that for every perfect matching $P$ of $Q_{d}$ there exists a walk between $P$ and $I$ in $\mathcal{M}\left(Q_{d}\right)$. Lemma 8 says that perfect matchings $\left[I_{d}\right]$ belong to a common component of $\mathcal{M}\left(Q_{d}\right)$, so it is sufficient to find a walk from $P$ to an arbitrary one of $\left[I_{d}\right]$. Without loss of generality we assume that $P$ is odd by Theorems 1 and 5 . We find this walk in two steps: First, we find a walk from $P$ to some perfect matching of $\mathcal{Z}(d, k, \alpha)$ for some $\alpha \in[d]$ and $k, d \geq k \geq 3$. Next, for every perfect matching of $\mathcal{Z}(d, k, \alpha)$ we find a walk to some perfect matching of $\mathcal{Z}(d, k+1, \alpha)$, so by induction on $k$ we obtain a walk from $P$ to $\mathcal{Z}(d, d, \alpha)$ which contains only $I_{d}^{\alpha}$ by definition.

Since $Q_{d}$ is bipartite, we call vertices of one color class black and the other white.

Lemma 11. For every odd perfect matching $P$ of $B\left(Q_{d}\right)$ there exists $Y \in \mathcal{Z}(d, k, \alpha)$ for some dimension $\alpha \in[d]$ and $k, d \geq k \geq 3$, such that $d(P, Y) \leq 3$.

Proof. We prove by induction on $d$ that for every perfect matching $P$ of $B\left(Q_{d}\right)$ there exist perfect matchings $R, X$ and $Y$ of $Q_{d}$ such that $P \cup R, R \cup X$ and $X \cup Y$ are Hamiltonian cycles and $X$ crosses $[d] \backslash\{\alpha\}$ and $Y \in \mathcal{Z}(d, k, \alpha)$.

First, we prove the statement for $d=3$. Let $P$ be an odd perfect matching of $B\left(Q_{3}\right)$. Therefore, $c\left(P \cup I_{3}^{\delta}\right)$ is 1 or 3 for every $\delta \in[3]$. If there exists $\delta \in[3]$ such that $c\left(P \cup I_{3}^{\delta}\right)=1$, then we choose $R:=Y:=I_{3}^{\delta}$ and $X \in \Gamma(R)$.

We prove that there exists $\delta \in[3]$ such that $c\left(P \cup I_{3}^{\delta}\right)=1$. Suppose on the contrary that $c\left(P \cup I_{3}^{\delta}\right)=3$ for every $\delta \in[3]$. The graph on edges $P \cup I_{3}^{\delta}$ consists of two common edges and one cycle of size 4 . Perfect matchings of $\left[I_{3}\right]$ are pairwise disjoint and $P$ has two common edges with each of them. This is a contradiction because $P$ has only 4 edges.

In the induction step we need to have a dimension $\gamma \in[d]$ that is crossed by at least 4 edges of $P$. If $d \geq 5$, such a dimension exists for every perfect matching $P$ of $B\left(Q_{d}\right)$ by the pigeonhole principle. Every perfect matching $P$ of $B\left(Q_{4}\right)$ has 8 edges. If $P$ contains an edge crossing at least two dimensions, then we use the pigeonhole principle again.

A perfect matching $P$ of $Q_{4}$ is balanced if it has 2 edges in every dimension. Luckily, Kreweras [8] proved that there are 8 perfect matchings of $Q_{4}$ up to isomorphism and only two of them are balanced; see Figure 3. Check that the balanced perfect matchings $S_{4}^{3}$ drawn in Figure 2 and $R^{1}$ drawn of Figure 4 satisfy the requirements of this lemma.

Now, we present the induction step. Let $\gamma \in[d]$ such that $P$ has at least 4 edges crossing $\gamma$. Without loss of generality we assume that $\gamma=d$. We divide $Q_{d}$ into two $(d-1)$-subcubes $Q^{1}$ and $Q^{2}$ by dimension $\gamma$. Let $B^{i}:=B\left(Q^{i}\right)$ and $P^{i}:=P \cap E\left(B^{i}\right)$ for $i \in\{1,2\}$. Let $M$ be the set of vertices of $B^{1}$ that are uncovered by $P^{1}$. We know that $|M| \geq 4$. Moreover, $M$ has the same number of black vertices as white ones.

Let $b_{1}$ and $b_{2}$ be two different black vertices of $M$ and $w_{1}$ and $w_{2}$ be two different white vertices of $M$. Let $S^{\prime}$ be a matching of $B^{1}$ covering $M \backslash\left\{b_{1}, b_{2}, w_{1}, w_{2}\right\}$. We have two ways of 
extending $S^{\prime}$ to obtain a matching $S^{1}$ of $B^{1}$ covering $M$ : We can insert edges $\left\{b_{1} w_{1}, b_{2} w_{2}\right\}$ or $\left\{b_{1} w_{2}, b_{2} w_{1}\right\}$. Those two ways give us two perfect matchings $P^{1} \cup S^{1}$ of $B^{1}$ having different parity. Of course, we choose the way that gives us odd perfect matching $P^{1} \cup S^{1}$.

Let $R^{1}, X^{1}$ and $Y^{1}$ be perfect matchings of $Q^{1}$ given by induction $-\left(P^{1} \cup S^{1}\right) \cup R^{1}, R^{1} \cup X^{1}$ and $X^{1} \cup Y^{1}$ are Hamiltonian cycles of $B^{1}, X^{1}$ crosses $[d-1] \backslash\{\alpha\}$ and $Y^{1} \in \mathcal{Z}(d-1, k, \alpha)$. Hence, $R^{1}$ is even by Theorem 5 . Let $S^{2}$ be given by (1).

We prove that $P^{2} \cup S^{2}$ is odd. Let $\bar{R}^{2} \in \Gamma\left(P^{2} \cup S^{2}\right)$ by Theorem 1 . Let $\bar{R}:=R^{1} \cup \bar{R}^{2}$. By Lemma 3 it holds that $\bar{R} \in \Gamma(P)$, so $\bar{R}$ is even by Theorem 5 . Also $\bar{R}^{2}$ is even because $R^{1}$ and $\bar{R}$ are even. Hence, $P^{2} \cup S^{2}$ is odd by Theorem 5. Moreover, $P^{2} \cup S^{2} \neq I_{d-1}^{\alpha}$.

Hence, the perfect matching $P^{2} \cup S^{2}$ crosses some $\beta \in[d-1] \backslash\{\alpha\}$ and there exists $R^{2} \in \Gamma\left(P^{2} \cup S^{2}\right)$ avoiding $\beta$ by Lemma 4 . Let $R:=R^{1} \cup R^{2}$. Therefore, $R \in \Gamma(P)$ by Lemma 3 and $R$ is even by Theorem 5. Because $R^{1}$ is even, $R^{2}$ is even. We apply Lemma 7 on $R^{1}, X^{1}, Y^{1}$ and $R^{2}$ to obtain a perfect matching $X$ such that $\left\langle R^{1} \mid R^{2}\right\rangle \cup X$ and $X \cup\left\langle Y^{1} \mid R^{2}\right\rangle$ are Hamiltonian cycles of $Q_{d}$ and $X$ crosses $[d] \backslash\{\alpha\}$. Finally, $Y:=\left\langle Y^{1} \mid R^{2}\right\rangle \in \mathcal{Z}(d, k, \alpha)$ by definition.
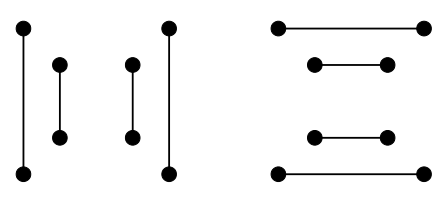

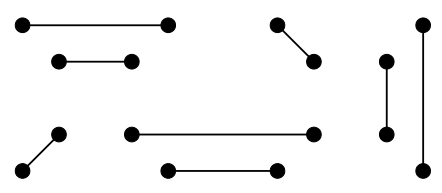

Perfect matching $R_{1}$

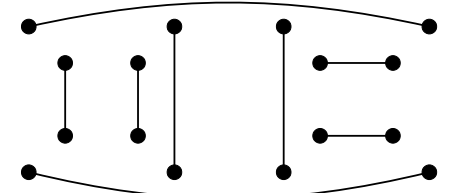

Perfect matching $R_{2}$

Perfect matching $R_{0}=P=\left[I_{3}^{\alpha} \mid I_{3}^{\gamma}\right]$

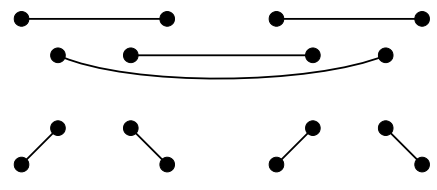

Perfect matching $R_{3}$

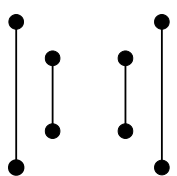

Perfect matching $R_{4}=I_{4}^{\alpha}=S$

Figure 4: A walk between $P \in \mathcal{Z}(4,3, \alpha)$ and $I_{4}^{\alpha}$.

Lemma 12. Let $P \in \mathcal{Z}(d, k, \alpha)$, where $3 \leq k<d$ and $\alpha \in[d]$. If $\mathcal{M}\left(Q_{k}\right)$ is connected or $k=3$, then there exists $S \in \mathcal{Z}(d, k+1, \alpha)$ such that $d(P, S)<\infty$.

Proof. We prove by induction on $d$ that for every $P \in \mathcal{Z}(d, k, \alpha)$ there exists a walk $P=$ $R_{0}, R_{1}, \ldots, R_{n}=S$ in $\mathcal{M}\left(Q_{d}\right)$ of even length such that $R_{l}$ crosses $[d] \backslash\{\alpha\}$ for every odd $l$ and $S \in \mathcal{Z}(d, k+1, \alpha)$. The base of this induction is for $d=k+1$.

By definition of $\mathcal{Z}(d, k, \alpha)$ we divide $P$ into perfect matchings $P^{1}$ and $P^{2}$ such that $P=$ $\left\langle P^{1} \mid P^{2}\right\rangle, P^{1} \in \mathcal{Z}(d-1, k, \alpha)$ and $P^{2}$ is an even perfect matching of $Q_{d-1}$ avoiding some $\beta \in[d-1] \backslash\{\alpha\}$.

First, we present the base of induction for $d=4$, so $k=3$. By definition, $P^{1}=I_{3}^{\alpha}$ and $P^{2}$ is even. There are two perfect matchings of $Q_{3}$ up to isomorphism with different parities; see Figure 1. Hence, $P^{2}=I_{3}^{\gamma}$ for some $\gamma \in[3]$. If $P^{2}=I_{3}^{\alpha}$, then $P=I_{4}^{\alpha}$, which belongs to $\mathcal{Z}(4,4, \alpha)$ by definition. Otherwise, the walk in Figure 4 satisfies requirements of this lemma.

Now, we present the base of the induction for $k \geq 4$ and $k+1=d$. In that case $P^{1}=I_{k}^{\alpha}$. There exists a walk $P^{2}=R_{0}, R_{1}, \ldots, R_{n}=I_{k}^{\alpha}$ on $\mathcal{M}\left(Q_{k}\right)$ of even length because 
$\mathcal{M}\left(Q_{k}\right)$ is connected and bipartite and $P^{2}$ is even. Let $R_{l}^{\prime}:=\left\langle P^{1} \mid R_{l}\right\rangle$ for even $l$. Clearly, $R_{n}^{\prime} \in \mathcal{Z}(d, k+1, \alpha)$ because $R_{n}^{\prime}=I_{k+1}^{\alpha}$.

Let $l$ be odd. Since $R_{l}$ is odd, it holds that $R_{l} \neq I_{k}^{\alpha}$. We choose an edge $e_{l} \in R_{l} \backslash I_{k}^{\alpha}$. By Proposition 2 there exists $Z_{l} \in \Gamma\left(I_{k}^{\alpha}\right)$ containing $e_{l}$. The perfect matching $Z_{l}$ crosses $[k] \backslash\{\alpha\}$ by Lemma 4 . We apply Lemma 6 on $R_{l-1}, R_{l}, R_{l+1}, I_{k}^{\alpha}, Z_{l}$, and $I_{k}^{\alpha}$ to obtain a perfect matching $R_{l}^{\prime}$. The walk $P=R_{0}^{\prime}, R_{1}^{\prime}, \ldots, R_{n}^{\prime}=I_{k+1}^{\alpha}$ satisfies the requirements.

Finally, we present the induction step for $k \geq 3$ and $k+1<d$. By induction there exists a walk $P^{1}=R_{0}, R_{1}, \ldots, R_{n}=S^{1}$ in $\mathcal{M}\left(Q_{d-1}\right)$ of even length such that $S^{1} \in \mathcal{Z}(d-1, k+1, \alpha)$ and $R_{l}$ crosses $[d-1] \backslash\{\alpha\}$ for every odd $l$. Let $R_{l}^{\prime}:=\left\langle R_{l} \mid P^{2}\right\rangle$ for even $l$. For odd $l$ we apply Lemma 7 on $R_{l-1}, R_{l}, R_{l+1}$ and $P^{2}$ to obtain a perfect matching $R_{l}^{\prime}$ of $Q_{d}$ crossing $[d] \backslash\{\alpha\}$. Now, the walk $P=R_{0}^{\prime}, R_{1}^{\prime}, \ldots, R_{n}^{\prime}=S$ satisfies the requirements and $S \in \mathcal{Z}(d, k+1, \alpha)$.

Corollary 13. Let $P \in \mathcal{Z}(d, k, \alpha)$, where $3 \leq k \leq d$ and $\alpha \in[d]$. If $\mathcal{M}\left(Q_{l}\right)$ is connected for every $l \in\{4,5, \ldots, d-1\}$, then $d\left(P, I_{d}^{\alpha}\right)<\infty$.

Proof. The proof proceeds by induction on $d-k$. If $d=k$, then $P=I_{d}^{\alpha}$ by definition of $\mathcal{Z}(d, k, \alpha)$. Let $3 \leq k<d$. By Lemma 12 there exists $S \in \mathcal{Z}(d, k+1, \alpha)$ such that $d(P, S)<\infty$. By induction $d\left(S, I_{d}^{\alpha}\right)<\infty$. Hence, $d\left(P, I_{d}^{\alpha}\right)<\infty$.

Theorem 14. The matching graph $\mathcal{M}\left(Q_{d}\right)$ is connected for $d \geq 4$.

Proof. The proof proceeds by induction on $d$. Kreweras [8] proved that the graph $M_{4}$ is connected; see Figure 3. Hence, the graph $\mathcal{M}\left(Q_{4}\right)$ is connected by Proposition 9 and the statement holds for $d=4$. Let us assume that the graph $\mathcal{M}\left(Q_{l}\right)$ is connected for every $l$ with $4 \leq l \leq d-1$. Let us prove that for some $\beta \in[d]$ and for every perfect matching $P$ of $Q_{d}$ it holds that $d\left(P, I_{d}^{\beta}\right)<\infty$.

If $P$ is even, then we choose $R \in \Gamma(P)$ by Theorem 1 which is odd by Theorem 5 . Otherwise, we simply consider $R:=P$. By Lemma 11 there exists $S \in \mathcal{Z}(d, k, \alpha)$ such that $d(R, S) \leq 3$. By Corollary 13 it holds that $d\left(S, I_{d}^{\alpha}\right)<\infty$ and $d\left(I_{d}^{\alpha}, I_{d}^{\beta}\right) \leq 6$ by Lemma 8 .

Corollary 15. The graph $M_{d}$ is connected for $d \geq 3$.

Acknowledgements. I am very grateful to Petr Gregor, Václav Koubek and Tomáš Dvořák for fruitful discussions on this topic.

\section{References}

[1] D. Dimitrov, T. Dvořák, P. Gregor, and R. Škrekovski. Gray codes faulting matchings. Submitted.

[2] R. J. Douglas. Bounds on the number of hamiltonian circuits in the n-cube. Discrete Math., 17:143-146, 1977.

[3] T. Dvořák. Hamiltonian cycles with prescribed edges in hypercubes. SIAM J. Discret. Math., 19(1):135-144, 2005.

[4] J. Fink. Matching graphs of hypercubes and complete bipartite graphs. In Proceedings of European Conference on Combinatorics, Graph Theory and Applications (EuroComb 2007), volume 29 of Electronic Notes in Discrete Mathematics, pages 345-351, 2007. 
[5] J. Fink. Perfect matchings extend to hamilton cycles in hypercubes. J. Comb. Theory, Ser. B, 97(6):1074-1076, 2007.

[6] P. Gregor. Perfect matchings extending on subcubes to hamiltonian cycles of hypercubes. DOI: 10.1016/j.disc.2008.02.013, 2008.

[7] L. Gros. Théorie du Baguenodier. Aimé Vingtrinier, Lyon, 1872.

[8] G. Kreweras. Matchings and hamiltonian cycles on hypercubes. Bull. Inst. Combin. Appl., 16:87-91, 1996.

[9] F. Ruskey and C.D. Savage. Hamilton cycles that extend transposition matchings in cayley graphs of $s_{n}$. SIAM Journal on Discrete Mathematics, 6(1):152-166, 1993.

[10] C. Savage. A survey of combinatorial gray codes. SIAM Review, 39(4):605-629, 1997. 